\title{
High-resolution UV relay lens for particle size distribution measurements using holography
}

\author{
Robert M. Malone**a ${ }^{\mathrm{a}}$ Gene A. Capelle ${ }^{\mathrm{b}}$, Brent C. Frogget ${ }^{\mathrm{a}}$, Mike Grover ${ }^{\mathrm{b}}$, Morris I. Kaufman ${ }^{\mathrm{a}}$, \\ Peter Pazuchanics ${ }^{c}$, Danny S. Sorenson ${ }^{c}$, Gerald D. Stevens ${ }^{b}$, Aric Tibbitts ${ }^{a}$, William D. Turley \\ ${ }^{a}$ National Security Technologies, LLC, Los Alamos Operations, \\ 182 East Gate Dr., Los Alamos, NM 87544 \\ ${ }^{\mathrm{b}}$ National Security Technologies, LLC, Special Technologies Laboratory, \\ 5540 Ekwil St., Santa Barbara, CA 93111 \\ 'Los Alamos National Laboratory, P. O. Box 1663, Los Alamos, NM 87545
}

\begin{abstract}
Shock waves passing through a metal sample can produce ejecta particulates at a metal-vacuum interface. Holography records particle size distributions by using a high-power, short-pulse laser to freeze particle motion. The sizes of the ejecta particles are recorded using an in-line Fraunhofer holography technique. Because the holographic plate would be destroyed in an energetic environment, a high-resolution lens has been designed to relay the interference fringes to a safe environment. Particle sizes within a 12-mm-diameter, 5-mm-thick volume are recorded onto holographic film. To achieve resolution down to $0.5 \mu \mathrm{m}$, ultraviolet laser (UV) light is needed. The design and assembly of a nine-element lens that achieves $>2000 \mathrm{lp} / \mathrm{mm}$ resolution and operates at $\mathrm{f} / 0.89$ will be described. To set up this lens system, a doublet lens is temporarily attached that enables operation with $532-\mathrm{nm}$ laser light and $1100 \mathrm{lp} / \mathrm{mm}$ resolution. Thus, the setup and alignment are performed with green light, but the dynamic recording is done with UV light. During setup, the 532-nm beam provides enough focus shift to accommodate the placement of a resolution target outside the ejecta volume; this resolution target does not interfere with the calibrated wires and pegs surrounding the ejecta volume. A television microscope archives images of resolution patterns that prove that the calibration wires, interference filter, holographic plate, and relay lenses are in their correct positions. Part of this lens is under vacuum, at the point where the laser illumination passes through a focus. Alignment and tolerancing of this high-resolution lens will be presented, and resolution variation through the 5-mm depth of field will be discussed.
\end{abstract}

Keywords: UV optical relay, holography, submicron optical resolution, ejecta, particle size distribution

\section{INTRODUCTION}

Metals, when under shock-loaded conditions, can create complex ejecta phenomena depending on the properties of the material and the initial shock conditions. We are interested in particle ejection that results from a shock wave interacting with at a metal-vacuum interface. For these experiments, particle sizes are measured using a holographic technique. Figure 1 shows examples of possible ejecta conditions on a metal surface. We will measure microjet production from grooves that are machined into the surface of a metal. The energy in the microjets will depend on the initial groove angle

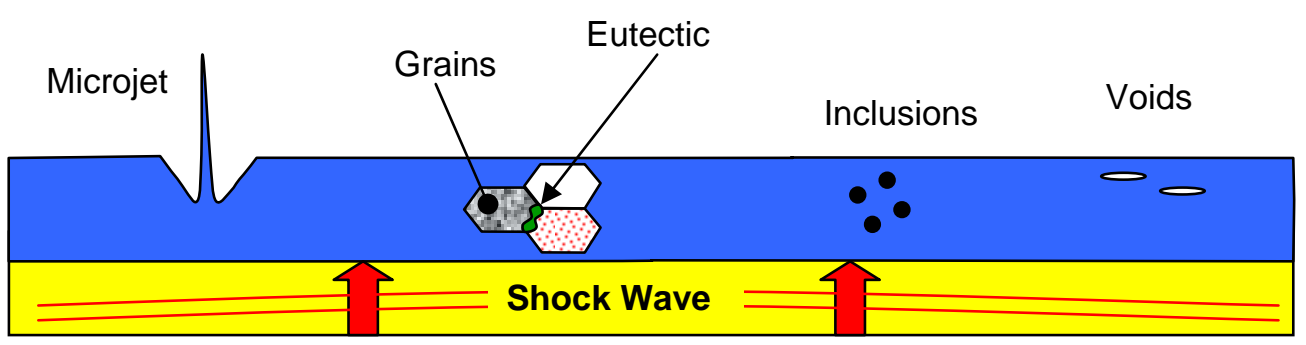

Fig.1. Illustration of possible ejecta formation processes.

*malonerm@nv.doe.gov, phone 1-505-663-2014; fax 1-505-663-2003 


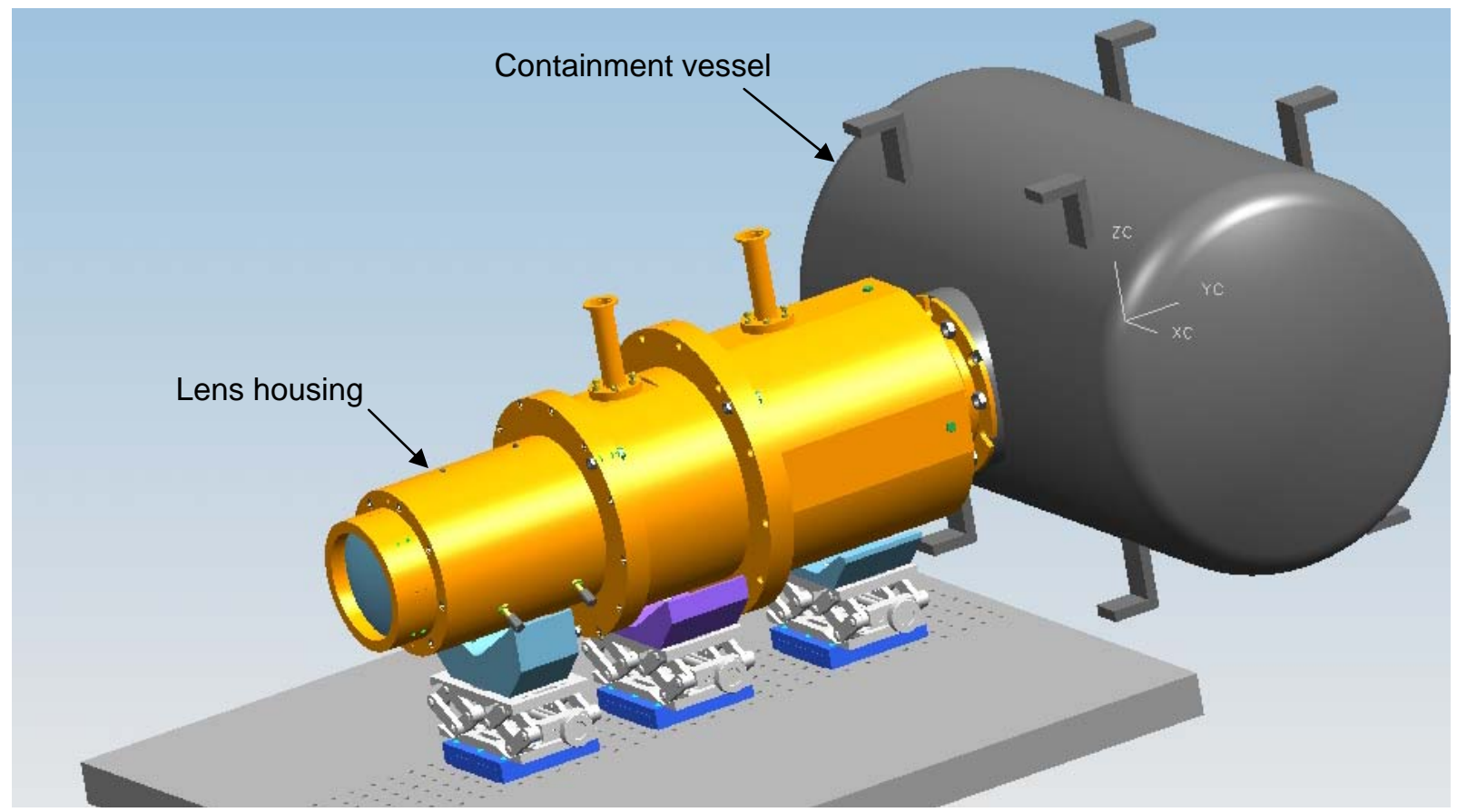

Fig.2. UV lens housing mated up to containment vessel (hologram holder not shown). Laser light travels from right to left. The lens housing (shown in yellow) is 31 inches long.

that is machined into the target material. One benefit of holography is that with a short-pulse laser, it can capture a threedimensional (3-D) image of high-speed ejecta. In particular, the in-line Fraunhofer holography technique was chosen because only one laser beam is required to make the measurement. ${ }^{1,2,3}$

An in-line holographic imaging system was developed and used for hydrodynamic experiments at the Pegasus facility (which no longer exists) located at Los Alamos National Laboratory. ${ }^{2,3}$ Holography offers the unique capability to record distributions of particles over a 3-D volume. We applied a holographic technique to measure ejecta particle size distributions for shock-loaded Sn over phase space that covers well below melt, near melt, and well above melt conditions. Pressures were 150 kbar, 400 kbar, and 700 kbar. Grooves with opening angles of 60, 90, and 120 degrees were machined into the target. These gave well-characterized initial conditions for the formation of the microjets. The amount of energy available for the fragmentation process increases for the smaller angle grooves. We used this data to compare with model predictions. This earlier work used a relay lens with $1000 \mathrm{lp} / \mathrm{mm}$ resolution recorded with a doubled Nd:YAG laser. We were able to measure particle sizes down to $1 \mu \mathrm{m}$.

Holography records particle size distributions by using a high-power, short-pulse laser to freeze particle motion. A new high-resolution lens (>2000 lp/mm resolution, operating at f/0.89) has been designed to relay the holographic interference fringes out of an energetic environment and into a protected holographic film can. Particle sizes within a 12-mm-diameter, 5-mm-thick volume are recorded onto the holographic film. To achieve resolution down to $0.5 \mu \mathrm{m}$, UV laser light is needed. To set up this lens system, a doublet lens is temporarily attached that enables operation with 532-nm laser light. Part of this lens is under vacuum, at the point where the laser illumination passes through a focus. Figure 2 shows how our lens will be mated to a containment vessel to conduct measurements on ejecta. The holographic film can and interference filter are to the left of the lens and are not shown. Part of the lens also extends inside the vessel.

\section{OPTICAL DESIGN}

The in-line holographic technique dictates that the object and reference beams use the same path to the holographic plate. One constraint is that there is $>95 \%$ transmission through the region to be recorded. There must be enough unscattered, undiffracted light for the reference beam to make good interference fringes with the ejecta particles. Immediately outside the ejecta region, these two beams form interference fringes. However, we cannot put the 
holographic plate in this destructive environment. So, a high-resolution relay system transfers the interference fringes to a safe environment.

High-resolution holographic film is not very light-sensitive. The system uses $>50 \mathrm{~mJ}$ of energy from the 150 -ps pulse duration laser sources. This laser light is collimated when passing through the ejecta region. This collimated light will focus at the stop position located in the middle of the lens system. Unless this location is under vacuum, the air will break down and destroy the reference beam wave front. A $>10^{-4}$ Torr vacuum at this location will preserve reference beam quality.

Earlier attempts at collecting particle size distributions onto holograms used a high-resolution lens with $1000 \mathrm{lp} / \mathrm{mm}$ resolution. ${ }^{3}$ A 532-nm pulsed laser exposed the hologram with $50 \mathrm{~mJ}$ of energy in $80 \mathrm{ps}$. This high-resolution lens consisted of two lens groups, each with five elements that required precision alignment. A 15-mm-diameter by 6-mmthick cylindrical volume of ejecta was recorded onto 62-mm-square holographic film. This lens had a magnification of $4 \mathrm{X}$ and collected light at $\mathrm{f} / 1.21$. Requirements for future dynamic shock experiments called for $2000 \mathrm{lp} / \mathrm{mm}$ resolution to measure down to a $0.5-\mu \mathrm{m}$ particle size. This resolution required use of a tripled Nd:YAG laser and a much lower f/\#.

The final lens configuration was achieved through the $\mathrm{CodeV}^{4}$ lens design program. The CodeV global optimization feature was used extensively to develop the lens system design. We started with a seven-element lens and gradually increased the number of lenses and their diameters until the required resolution was achieved. When selecting the best lens form factor after each global optimization run, we were diligent in achieving minimum ray bending at each lens surface. Minimum ray bending between these surfaces relaxes the tolerance requirements.

The final UV lens system, shown in Figure 3, uses nine elements. Only after allowing both the image and the object planes to be curved were we able to get to the desired resolution. It is more important to achieve high resolution than it is to know the exact position of the ejecta particle. So, the object and image planes were allowed to be curved. This is unusual because most imaging applications require flat image planes.

This optical design is a one-sided telecentric system, leaving the holographic plate side non-telecentric. The approach to correcting 1st order longitudinal chromatic aberration is to use crown glass for the positive elements and flint glass for negative ones. However, using higher index flint glass for one of the positive lenses reduced the largest lens's diameter.

The ejecta data measure $12 \mathrm{~mm}$ in diameter by $6 \mathrm{~mm}$ thick. Because of the lens magnification, the image of these particles (relayed ejecta region) measures $60 \mathrm{~mm}$ in diameter by $125 \mathrm{~mm}$ thick. The laser focus is protected inside a vacuum chamber. Because the sticky holographic emulsion can attract dirt particles, the relayed ejecta data must be positioned well away from any contaminants. An interference filter is also placed outside the relayed ejecta region. This filter blocks white light from the detonator that accelerates the target material.

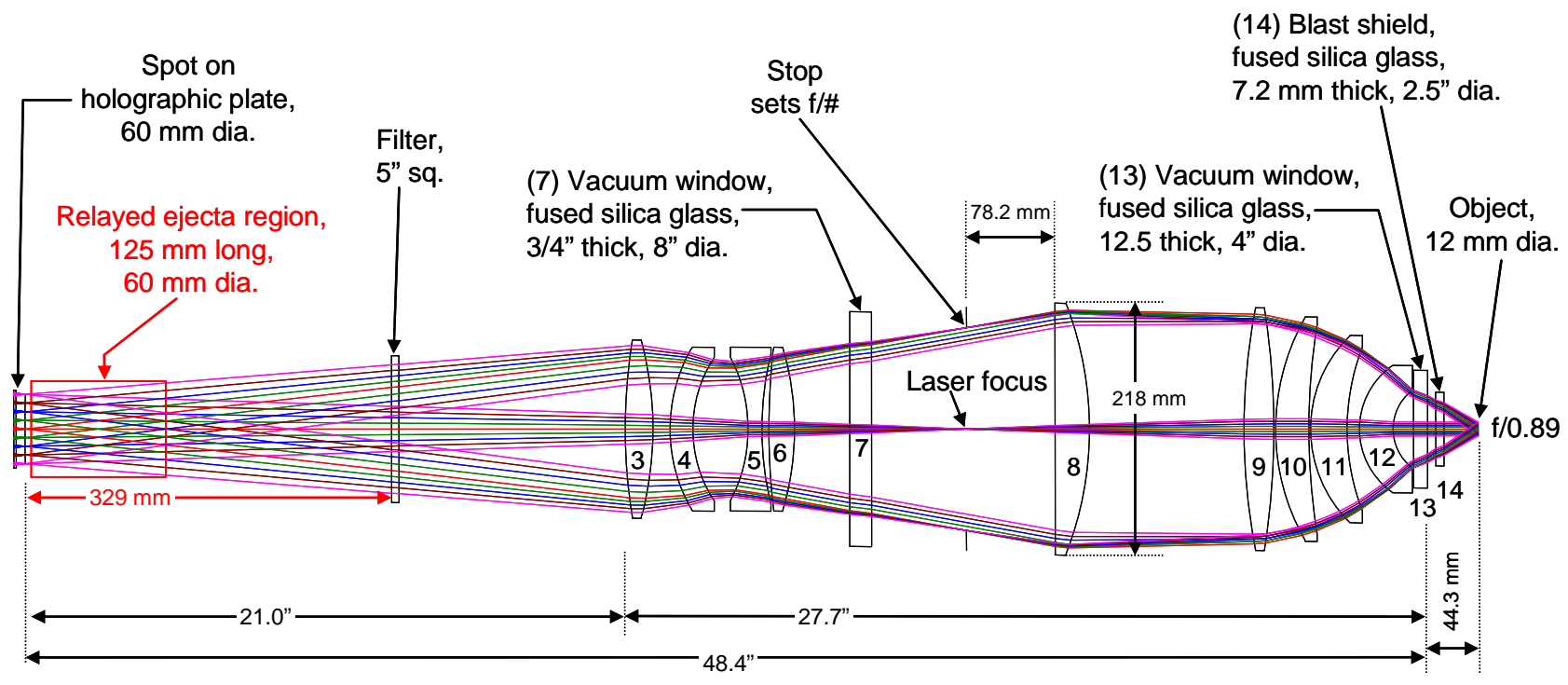

Fig. 3. The holographic lens for recording with 354.7-nm laser light uses nine lenses, providing 5X magnification. Laser light travels from right to left. 


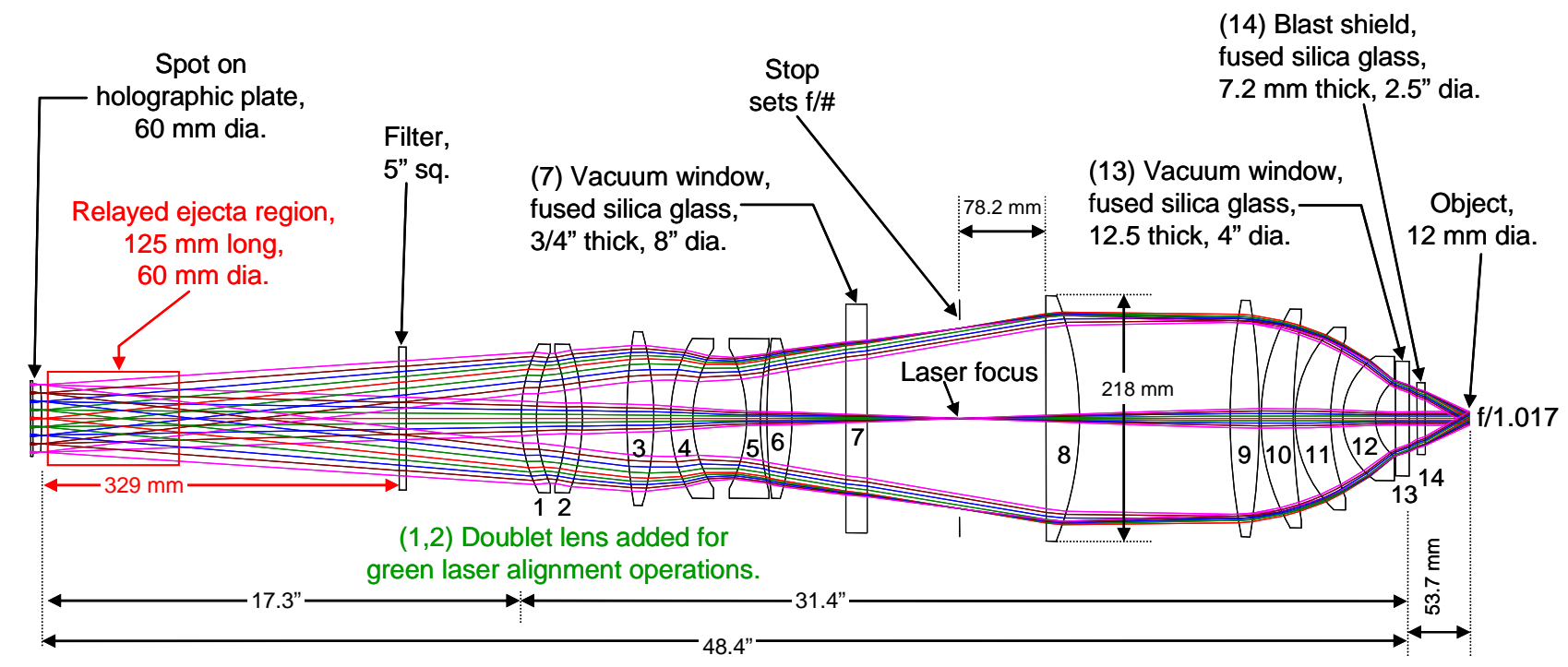

Fig. 4. The holographic lens for recording with 532-nm laser light uses eleven lenses, providing 5X magnification.

It is difficult to work with UV light during lens system setup. To address the fact that UV light poses an eye hazard, a doublet lens was designed to be mounted to the UV lens system. This configuration allows alignment work to be performed at $532 \mathrm{~nm}$ (green); see Figure 4. The doublet is pinned to the larger lens housing to allow for repeatable alignment. One constraint is that the position of the hologram is fixed with or without the doublet lens. The result causes a shift in the object plane where the ejecta are created. We will only measure $1100 \mathrm{lp} / \mathrm{mm}$ resolution with this green laser mode, but experiment set up and documentation are easily facilitated.

All lens and window surfaces have a double V-coating at both 354 and $532 \mathrm{~nm}$, except for two lenses (elements 1 and 2) that only require a V-coating at $532 \mathrm{~nm}$ on their surfaces. All coatings are $<0.1 \%$ reflective at the 0 -degree angle of incidence. The laser damage threshold for these coatings is $0.2 \mathrm{~J} / \mathrm{sq}$. cm with a 5-ns laser pulse at $354 \mathrm{~nm}$. All coatings are for random polarizations.

The resolution performance of the dual modes is shown in Figure 5. The UV mode is very nearly diffraction limited over the full field of view. The holographic film has better than $3000 \mathrm{lp} / \mathrm{mm}$ resolution. With the $5 \mathrm{X}$ magnification, the film needs to record at only $400 \mathrm{lp} / \mathrm{mm}$ resolution.

Since chromatic aberrations do not exist for the single wavelength lasers used, only a few glass types are needed. The glasses used for the UV system were SBSL7 and PBM18Y from Ohara. These glasses were selected because of their transmission properties and cost. The UV lens was optimized by testing only these two glasses at each lens position. The glasses used for the green doublet were NBK7 and SF11 from Schott. The air spacings for the lens housing were recomputed after melt data, radius of curvature data, and lens element thickness were measured following the lens element fabrications.

\section{OPTICAL SETUP}

Earlier holographic work required taking multiple holographic exposures to align the optical system and correctly position the ejecta region (which included calibration wires and pegs) relative to the holographic film. This earlier procedure was very time consuming and drove up the cost of fielding holographic experiments. Now, a 6.6-megapixel CCD camera views data presented to the holographic plate. This camera has a 16X optical zoom lens with a 190-mm standoff distance. This CCD camera system is mounted on tip/tilt and translation stages. The translation stage allows focus shifts of $200 \mathrm{~mm}$ along the optical axis. We focus on the emulsion of the holographic plate and measure its position. We then shift the focus and measure the position of a calibration wire. This arrangement allows us to document the performance of the optical setup prior to the dynamic experiment. 


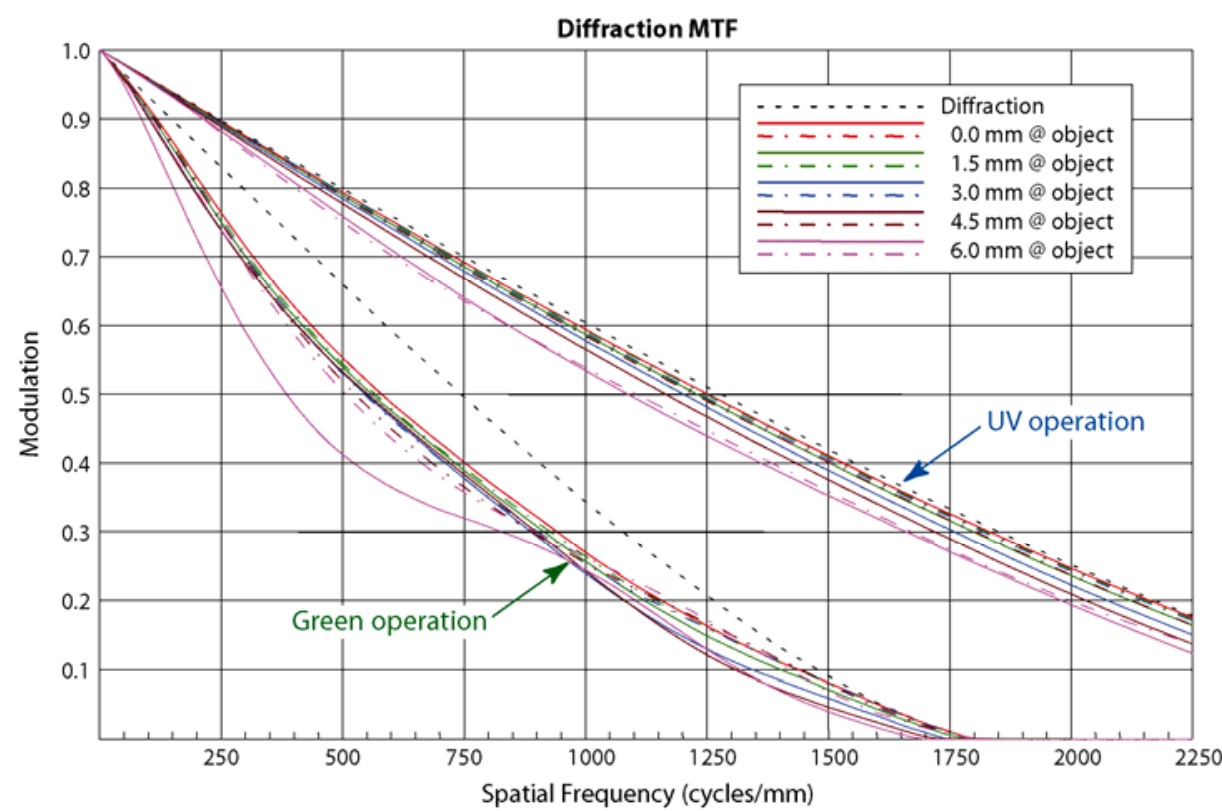

Fig.5. Resolution performance with and without the doublet lens. This resolution is measured at the object plane where the ejecta are created.

The light source for the CCD camera system is a low-power continuous wave (CW) doubled Nd:YAG laser. It is difficult to view the small features of the resolution target with this laser light because of laser speckle. Therefore, we place a spinning diffuser in front of the optical system. The diffuser has about a 5-degree spread. The spinning diffuser completely averages out the laser speckle. It allows us to find the best focus of the resolution target and the calibration wires. The spin rate is not critical.

With the lens system in place, there is considerable focus shift when viewing with 532-nm or 354.7-nm laser light. The position of the holographic film is fixed relative to the lens housing and the position of the resolution pattern placed in the ejecta region will shift by $9.4 \mathrm{~mm}$ depending on the wavelength used, as shown in Figure 6. The thickness of the ejecta region is $5 \mathrm{~mm}$. Therefore, a resolution target can be placed outside the delicate calibration wires and alignment pegs that define the ejecta region. We can document the image quality and alignment with the 532-nm laser and have confidence that the UV laser operation will function properly. The resolution target is removed before the dynamic event.

Counter-propagating CW alignment lasers (green and red) are used to set up the optical system. Apertures are also mounted to the front and back lens-housing flanges to establish the optical axis. Floating apertures are used several feet before and after the lens system to precisely detect alignment errors demonstrated by the laser spot groupings. Each aperture will show a grouping of spots in two colors, the result of either steering or retroflection errors. Great care is taken to keep the green and red lasers collinear to each other. The red laser is centered on the green laser's output mirror and vice versa.

\section{OPTOMECHANICAL DESIGN}

The housing shows two pump-out vacuum ports for faster pump out, but they are tied to only one vacuum pump. Only the stop aperture was black anodized with a vacuum-compatible inorganic black dye. To prevent corrosion to the aluminum, a chromate conversion coating was applied to all other metal surfaces. Nonfunctional threads are used on two of the air spacers to reduce unwanted light scatter. However, because the threads do not catch the light as well as expected, other techniques should be considered.

An extra-thick (0.5-inch) vacuum window is mounted to the front of the lens housing to protect the lens elements from possible damage by flying shrapnel during the dynamic event. The vacuum window is replaced after every event. 


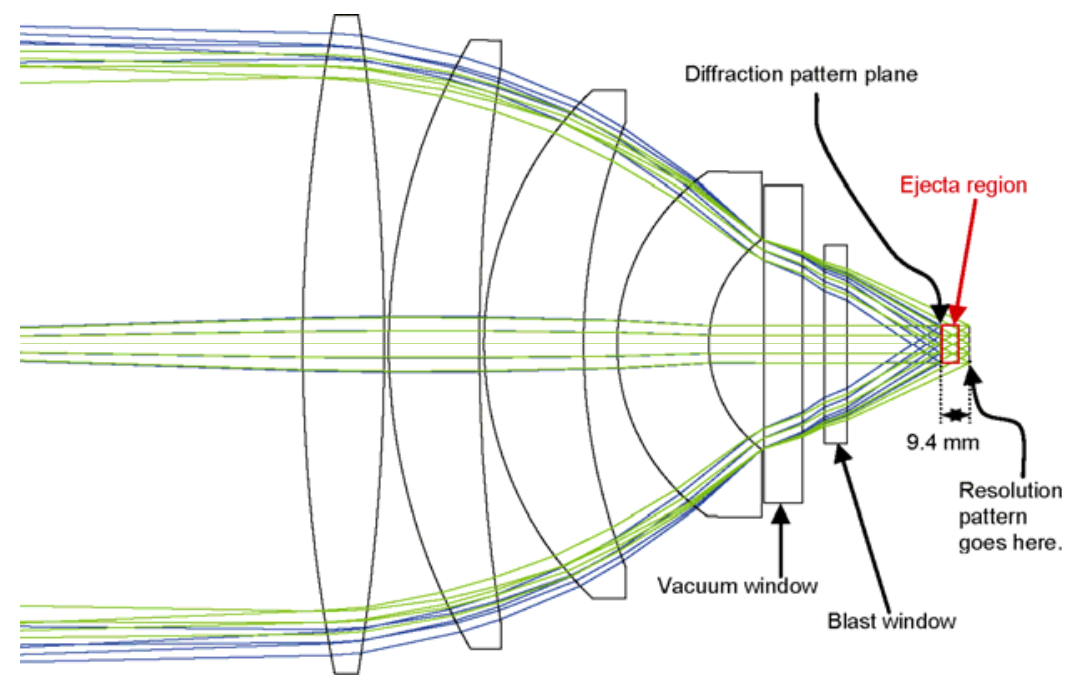

Fig. 6. The diffraction pattern plane contains the interference patterns produced inside the ejecta region using the 354.7-nm laser light. This plane is relayed with the optical system to a position $12 \mathrm{~mm}$ from the holographic emulsion. When operating with 532-nm laser light, an interference target can be placed outside the ejecta region and simulate what the diffraction pattern plane would present to the hologram emulsion.

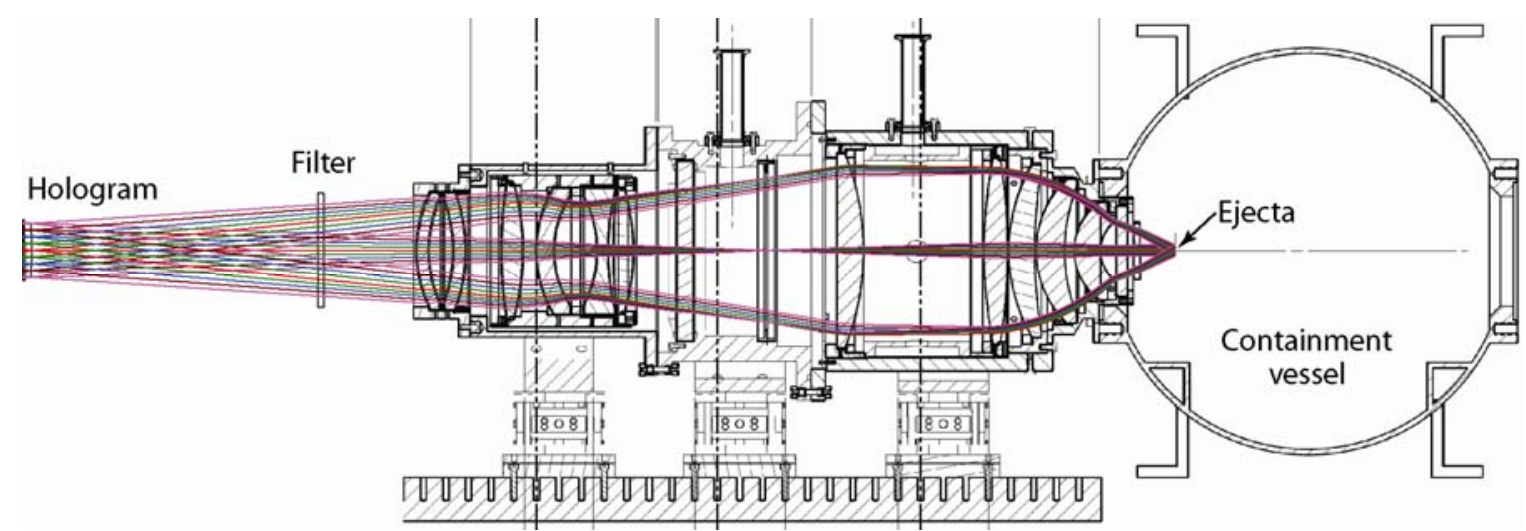

Fig. 7. The lens housing is mated up to the containment vessel. Laser light travels from right to left.

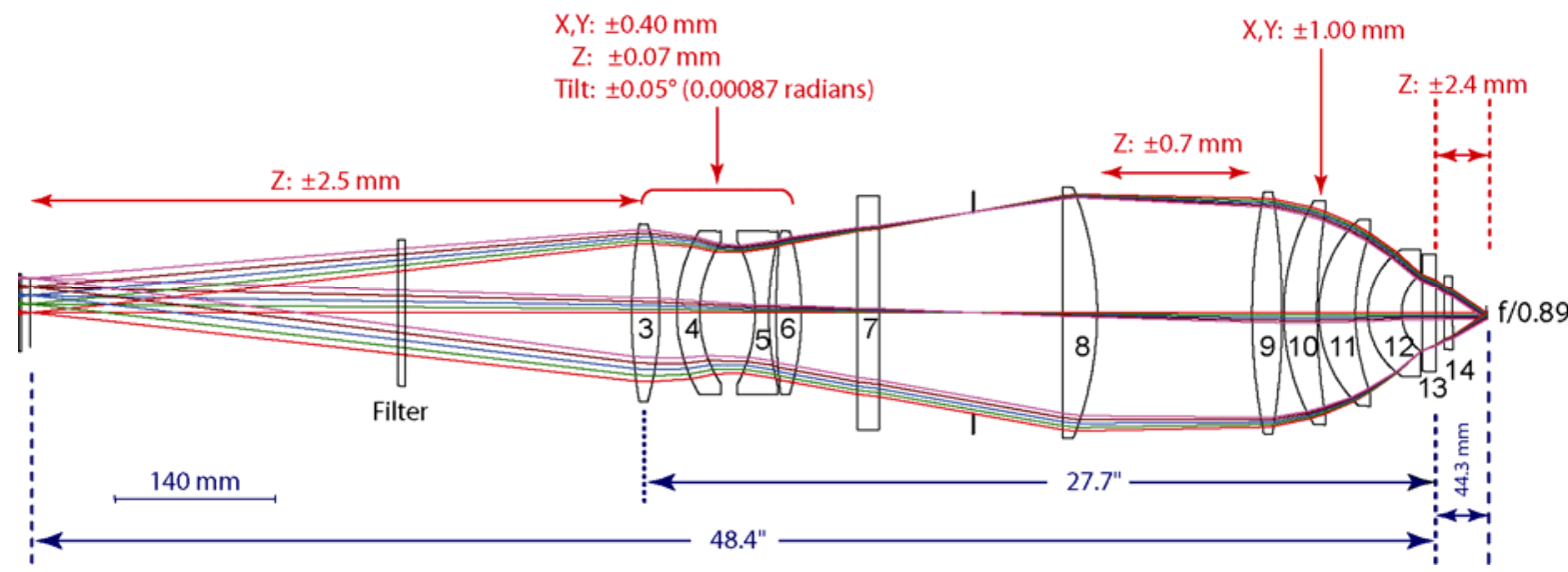

Fig. 8. Probable change of compensators (Monte Carlo analysis). Laser light travels from right to left. These compensator values were built into the metal housing adjustments. 
The lens housing, shown in Figure 7, is mated to the containment vessel with a thick flat gasket. The containment vessel is not under vacuum; only the dynamic package (not shown), where the ejecta are produced, and the lens housing are under vacuum. Shock generated during a dynamic event is a concern for the lens system. The support for the lens consists of three lab jacks mounted to dovetail rails. The lab jacks tilt the lens relative to the containment vessel. During a dynamic event, the rails allow the lens housing to move with the shock wave.

For shock mitigation, the retaining rings have rounded edges where they encounter a glass surface. An analysis of stresses on the metal-to-glass interface was done for up to $20 \mathrm{G}$ shock loading. We found that a $20-\mathrm{G}$ quasi-static load results in less than 1000 psi of stress on the glass near the rounded metal edges.

Initially the lens tolerances were very tight until we found the best set of compensators. After starting with all lenses inside the vacuum, we moved the lens subcell that contains elements 3, 4, 5, and 6 outside the vacuum where we could easily decenter and tilt them. We then found the lens element that was the most sensitive to decenter and made this a compensator. This lens (element 10) is inside the vacuum. So, after a one-time, single decentering is completed on this lens, its access holes will be sealed off with a pressure port O-ring screw. Figure 8 shows the location and values for the final compensators. We used the root sum of the squares (RSS) method to add up the most probable mechanical tolerance buildup. In most cases the RSS error buildup was $<75 \%$ of the error budget that was determined from a CodeV Monte Carlo error analysis for decentrations. Even though this lens system provides $2000 \mathrm{lp} / \mathrm{mm}$ resolution, whereas the earlier design provided $1000 \mathrm{lp} / \mathrm{mm}$, the housing tolerances were a factor of 5 looser.

To enhance the center-to-center alignment of this lens system, most of the lenses are mounted into subcells as shown in Figure 9. Care must be taken to not over tighten the centering screws on the lens cells before the glue operation. Over tightening these screws will make the lens cell out of round.

The total weight of the 14 elements used for the 532-nm alignment mode is $36 \mathrm{lbs}$. The total weight of the metal housing is 80 lbs.

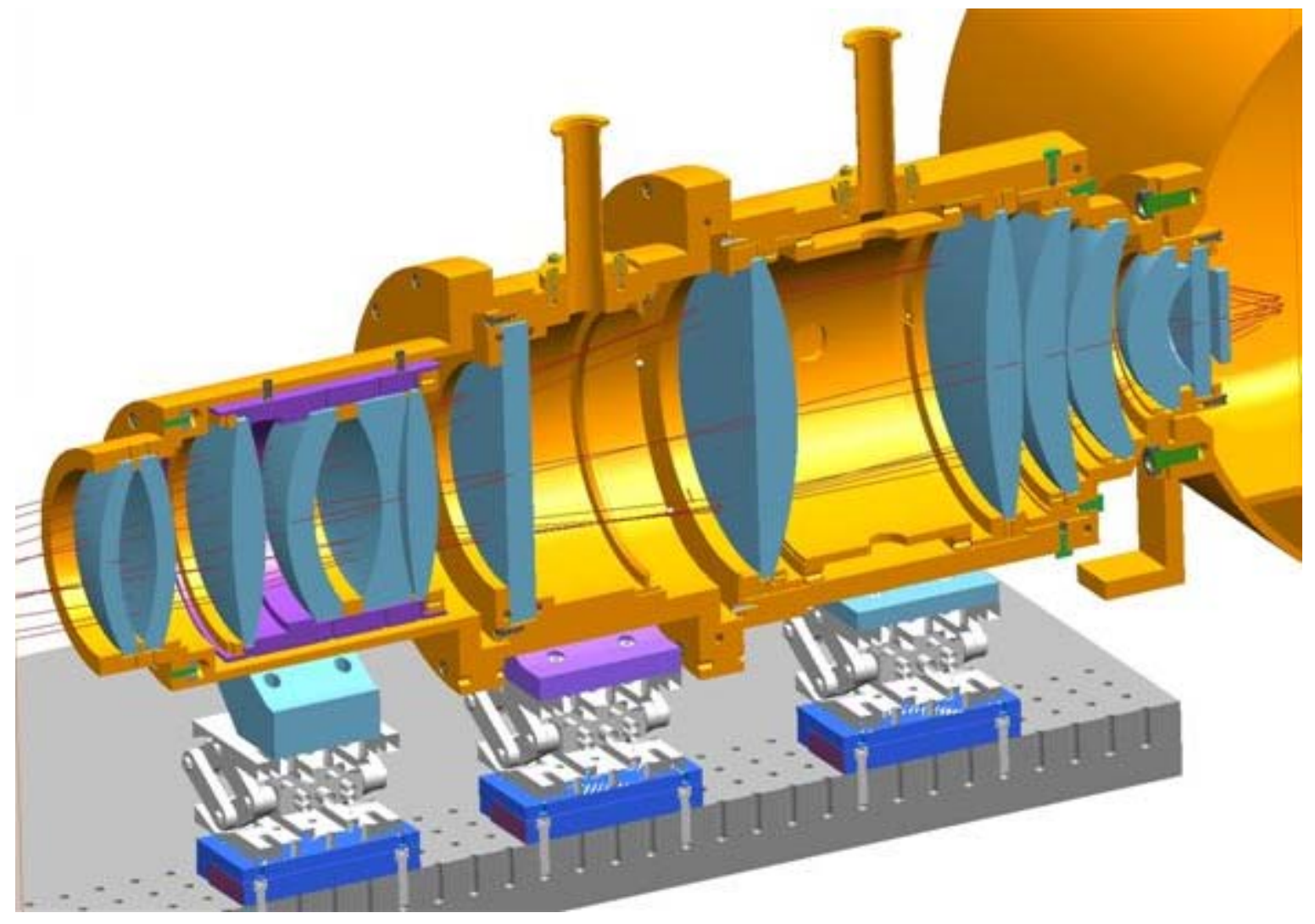

Fig. 9. Model of the lens housing. Laser light travels from right to left. 


\section{CONCLUSION}

Use of a CCD camera alignment system reduces the number of holograms that have to be taken before a dynamic event. We are able to document the optical system performance with digitized resolution patterns. Use of the CCD camera allows us to set up the optics more efficiently, which reduces fielding costs. Being able to set up with green laser light simplifies laser safety issues.

The amount of data recorded by the hologram is huge: recording $2000 \mathrm{lp} / \mathrm{mm}$ over a cylinder that measures $12 \mathrm{~mm}$ in diameter by $5 \mathrm{~mm}$ thick, collects 4.5 terabytes of data. An existing automated reconstruction system can process and store this data. ${ }^{5}$

\section{ACKNOWLEDGMENTS}

This manuscript has been authored by National Security Technologies, LLC, under Contract No. DE-AC52-06NA25946 with the U.S. Department of Energy. The United States Government retains and the publisher, by accepting the article for publication, acknowledges that the United States Government retains a non-exclusive, paid-up, irrevocable, worldwide license to publish or reproduce the published form of this manuscript, or allow others to do so, for United States Government purposes.

\section{REFERENCES}

[1] C. S. Vikram, Particle Field Holography, Cambridge Studies in Modern Optics (1992).

[2] D. S. Sorenson, R. M. Malone, B. C. Frogget, C. A. Ciarcia, T. W. Tunnell, R. L. Flurer, "Particle distribution measurements using in-line Fraunhofer holography,” Proc. SPIE 2869, 206-213 (1997).

[3] D. S. Sorenson, R. W. Minich, J. L. Romero, T. W. Tunnell, R. M. Malone, "Ejecta particle size distributions for shock loaded Sn and Al metals,” Journal of Applied Physics, 92, 5830-5836 (2002).

[4] CodeV is licensed software from Optical Research Associates, Pasadena, CA.

[5] T. W. Tunnell, R. M. Malone, R. H. Fredrickson, A. D. Delanoy, D. E. Johnson, C. A. Ciarcia, and D. S. Sorenson, “Deriving particle distributions from in-line Fraunhofer holographic data," Proc. SPIE 3163, 558-569 (1997). 